\title{
INCLUSIÓN DIFERENCIAL DE EXTRANJEROS/MIGRANTES A TRASPLANTES DE ÓRGANOS: DILEMAS ÉTICOS FRENTE A "PRÁCTICAS NO ÉTICAS"
}

\author{
Differential inclusion of foreigners / migrants to organ transplants: \\ ethical dilemmas in face of non-ethical practices
}

Lourdes Basualdo*

\begin{abstract}
Resumen. Este artículo aborda el modo en que ciertos dilemas éticos vinculados al trasplante de órganos a extranjeros "no residentes", son movilizados por el Estado argentino en las normativas que definen un tratamiento diferenciado entre nacionales y no-nacionales en materia de trasplante, y cómo son puestos en relación con la política migratoria. Se muestra que existen mecanismos de inclusión diferencial que garantizan la inscripción a lista de espera sólo a nacionales y extranjeros "residentes" y permiten el trasplante con donante vivo a extranjeros "no residentes". Estos procesos de apertura y cierre de fronteras son posibles porque en el contexto actual coexisten la preocupación estatal en resguardar los bienes sociales proporcionados por el Estado (una de las funciones constitutivas del control migratorio) y la obligación moral de los Estados-nación por el cuidado de la vida en el marco del gobierno humanitario.
\end{abstract}

Palabras claves: migración, ética, trasplantes de órganos, inclusión diferencial, Argentina.

\begin{abstract}
This article addresses the way in which certain ethical dilemmas related to organ transplantation to "nonresident" foreigners are enacted by the Argentine government through regulations that define differentiated treatments between national and non-national transplanted subjects. While establishing how they are connected to migration policies, it demonstrates that there are mechanisms of differential inclusion that guarantee the registration in waiting lists available only to nationals and foreign "residents" and allow live donor transplantation to "nonresident" foreigners. These processes of opening and closing of borders are possible because -in the current contextthere is a governmental concern with the protection of public goods provided by the State, one of the constituent functions of migration control. At the same time, there is a moral obligation of the Nation-state to life care within the framework of a humanitarian government.
\end{abstract}

Keywords: migration, ethics, organ transplantation, differential inclusion, Argentina.

\footnotetext{
* Consejo Nacional de Investigaciones Científicas y Técnicas (CONICET) - Centro de Investigaciones y Estudios sobre Cultura y Sociedad y Universidad Nacional de Córdoba. Córdoba, Argentina.
} 


\section{Introducción}

El acceso de personas extranjeras/migrantes a tratamientos de trasplantes de órganos, en particular aquellas en situación administrativa "irregular", constituye un tema controversial. Según la Organización Panamericana de la Salud, si bien los criterios de acceso a los servicios de trasplante varían de un país a otro, en pocos países se hace referencia a la posibilidad de que los extranjeros "no residentes" ingresen a la lista de espera para recibir órganos o tejidos donados y constituye una responsabilidad de cada Estado determinar bajo qué condiciones éstos podrán acceder a un trasplante ${ }^{1}$. En Argentina el Instituto Nacional Central Único de Ablación e Implante (INCUCAI), ente descentralizado y autárquico que depende de la Secretaría de Políticas, Regulación e Institutos del Ministerio de Salud de la Nación, y que regula la actividad de trasplantes en el país, indicó a través de una normativa específica que únicamente podrán inscribirse en lista de espera aquellos extranjeros que cuenten con residencia de carácter permanente.

El tratamiento diferenciado de nacionales y extranjeros/migrantes en lo que respecta a trasplante de órganos ha recibido escasa atención en la literatura académica. Particularmente en el contexto argentino, la sanción en el año 2004 de la ley de migraciones №25.871 que inauguró un periodo de cambio en el discurso estatal sobre las migraciones internacionales, basado en la retórica de la inclusión, en contraposición a la retórica de la exclusión imperante en los años noventa ${ }^{2}$, contribuyó a marcar un punto de inflexión en el interés científico, parte del cual buscó analizar la accesibilidad de los migrantes al sistema público de salud en diferentes lugares de Argentina. Algunos de los estudios desarrollados mostraron las condiciones que afectan el acceso a los servicios de salud de los inmigrantes de países limítrofes y del Perú ${ }^{3}$, el vínculo migración, salud y fronteras ${ }^{4}$, la situación de salud y la accesibilidad de las mujeres migrantes al sistema de salud ${ }^{5}$, y las diferentes formas que adquiere el acceso a la salud de

1 ORGANIZACIÓN PANAMERICANA DE LA SALUD. Legislación sobre donación y trasplante de órganos, tejidos y células: compilación y análisis comparado. Washington, D.C., 2013.

2 DOMENECH, Eduardo. La visión estatal sobre las migraciones en la Argentina reciente. De la retórica de la exclusión a la retórica de la inclusión.

3 ABEL, Lidia, CAGGIANO, Sergio. Enfermedades de estado(s). Los inmigrantes y el acceso a la salud en una provincia de frontera. In JELIN, Elizabeth. Salud y Migración Regional. Ciudadanía, y comunicación intercultural. Buenos Aires: IDES, 2006, p. 57-90; CAGGIANO, Sergio. 'Que se haga cargo su país': la cultura, los estados y el acceso a la salud de los inmigrantes bolivianos en Jujuy. In GARCÍA VÁZQUEZ, Cristina. Hegemonía e interculturalidad. Poblaciones originarias e inmigrantes. Buenos Aires: Prometeo, 2008, p. 243-279.

4 COURTIS, Corina, LIGUORI, Gabriela, CERRUTTI, Marcela. Migración y salud en zonas fronterizas. El Estado plurinacional de Bolivia y la Argentina. Santiago de Chile: CELADE, 2010.

5 CERRUTTI, Marcela. Salud y Migración Internacional: Mujeres Bolivianas en la Argentina. Buenos Aires: Latingráfica, 2010; CAGGIANO, Sergio. Madres en la frontera: género, nación y los peligros de la reproducción. Íconos. Revista de Ciencias Sociales, n. 27, 2007, p. 93-106; WANG, Lucía. Ideas y prácticas en torno a la salud sexual y reproductiva en jóvenes migrantes paraguayas que se trasladan 
los migrantes según el nivel de complejidad que requiera la atención, en donde la universalidad del acceso se reduce para los migrantes en situación "irregular" cuando las prácticas que demandan son $\operatorname{costosas}^{6}$. En líneas generales, varios de los estudios citados buscaron analizar la adecuación de la normativa migratoria a las políticas públicas de salud, cuestiones que, al ser observadas con relación a la ley migratoria anterior, identificaron avances y desafíos pendientes sobre el reconocimiento de derechos a los migrantes. Dentro de la variedad de temas abordados, el análisis de las normativas en materia de trasplantes y su vínculo con las migraciones ha estado ausente en las investigaciones mencionadas, salvo en los informes producidos por el Centro de Estudios Legales y Sociales ${ }^{7}$ que describen la existencia de resoluciones restrictivas al acceso de personas migrantes a prácticas trasplantológicas, pero no profundizan en los procesos de configuración de las medidas adoptadas.

Otros antecedentes que abordan específicamente la actividad de trasplante de órganos se han focalizado en los diferentes dilemas éticos que plantea el trasplante ${ }^{8}$, las desigualdades geográficas para el acceso al trasplante ${ }^{9}$, las formas en que las tecnologías interactúan con los $\operatorname{cuerpos}^{10}$, el papel del Estado en la distribución y la procuración de órganos ${ }^{11}$. Por otra parte, las discusiones sobre el valor comercial del cuerpo y de los órganos a partir de mediados de los años noventa, condujeron a plantear nuevos interrogantes de investigación, entre ellos: características del "comercio y el tráfico global de órganos" y el "turismo de trasplantes" a nivel transnacional ${ }^{12}$, la difusión internacional de la legislación sobre el "comercio y tráfico de órganos" y "turismo de trasplantes"13.

a la Argentina. Ea, v. 2, n. 2, 2010; AIZENBERG, Lila, RODRíGUEZ, María Laura, CARBONETTI, Adrian. Percepciones de los equipos de salud en torno a las mujeres migrantes bolivianas y peruanas en la ciudad de Córdoba. Migraciones Internacionales, v. VIII, n. 1, 2015, p. 65-94.

6 JELIN, Elizabeth. Salud y Migración Regional. Ciudadanía, discriminación y comunicación intercultural. Buenos Aires: IDES, 2006.

7 CELS. Estudio sobre los derechos de niños y niñas migrantes a 5 años de la nueva ley de migraciones. UNLa, Centro de Derechos Humanos, Programa y Derechos Humanos: Lanús, Argentina, 2010; CELS. Niñez, Migraciones y Derechos Humanos en Argentina. Estudio a diez años de la ley de migraciones. Lanús: Universidad Nacional de Lanús, Centro de Derechos Humanos, Programa Migraciones y Derechos Humanos, 2013.

8 IKELS, Charlotte. Ethical issues in organ procurement in Chinese societies. The China Journal, n. 38,1997, p. 95-119.

9 DAVIES, Gail. Patterning the geographies of organ transplantation: corporeality, generosity and justice. Transactions of the Institute of British Geographers, v. XXXI, n. 3, 2006, p. 257-271.

${ }^{10}$ HEINEMANN, Laura. Transplantation and Body Politics: Toward Assessing and Addressing Inequality. New Directions in Medical Anthropology, v. XVII, n. 1, 2008, p. 76-107.

11 PATERSON, Florence. Solliciter l'inconcevable ou le consentement des morts. Prélèvement d'organes, formes de circulation des greffons et normes de competence. Sciences sociales et santé, v. XV, n. 1, 1997, p. 35-74.

12 SCHEPER-HUGHES, Nancy. Las vacaciones del señor Tati y el safari africano de João: ver el mundo a través del turismo de trasplantes. Tráficos, v. II, n. 17, 2013, p. 77-105.

${ }^{13}$ AMAHAZION, Fikresus. Human rights and world culture: The diffusion of legislation against the organ trade. Sociological Spectrum, v. XXXVI, n. 3, 2016, p. 158-182. 
Las investigaciones citadas reconocen las desigualdades existentes en el acceso a la práctica trasplantológica a nivel internacional, pero la discusión sobre el rol del Estado en la definición de los criterios de distribución de órganos en función de su "maquinaria" administrativa de justicia estatal ${ }^{14}$ que condiciona el derecho de los extranjeros/migrantes al tratamiento, se ha encontrado ausente, salvo escasas excepciones que cuestionan en términos éticos y políticos por qué los inmigrantes "indocumentados" y solicitantes de asilo (o que han obtenido el estatuto de refugiados) no pueden acceder a un trasplante ${ }^{15}$. Tampoco ha sido indagada la manera que la categoría "turismo de trasplantes" se incorporó al discurso oficial de los organismos internacionales y diferentes Estados nacionales en la regulación del acceso al trasplante de la población extranjera/migrante.

El análisis que aquí se realiza, fundamentalmente a partir de la normativa migratoria y aquellas referidas a trasplante de órganos en Argentina, así como otras fuentes documentales oficiales relativas a la actividad de trasplante a nivel internacional, de comienzos de los años dos mil hasta la actualidad, propone identificar el modo en que ciertos dilemas éticos sobre trasplante a extranjeros/ migrantes, al ser puestos en relación con los contenidos relativos al derecho a la salud de la ley de migraciones argentina, generan mecanismos de inclusión diferencial a través de las normativas que regulan el acceso de la población no-nacional al tratamiento. En coincidencia con Mezzadra y Nielson el concepto de inclusión diferencial es útil para observar cómo la inclusión de los migrantes “puede estar sujeta a variantes grados de subordinación, reglas, discriminación y segmentación en un ámbito o esferas determinados"16 y permite analizar a las tensiones alrededor de las fronteras, en la medida en que éstas implican no sólo procesos de reforzamiento y bloqueo, sino también la necesidad de cruce por parte de las personas ${ }^{17}$. De acuerdo a lo anterior, este trabajo no persigue el objetivo de señalar desafíos pendientes relativos al cumplimiento del art. 8o de la actual ley de migraciones, el cual propone la igualdad en el acceso a la asistencia sanitaria por parte de los extranjeros cualquiera sea su situación administrativa migratoria. Ello implicaría asumir una discusión sobre los procesos de cierre y apertura de fronteras en términos de contradicción entre, por ejemplo, principios de ética liberal (en defensa de derechos humanos universales) y principios de ética comunitarista (como fundamento de los intereses y derechos de aquellos reconocidos como ciudadanos por el Estado).

\footnotetext{
${ }^{14}$ MEZZADRA, Sandro, NIELSON, Brett. Fronteras de Inclusión diferencial. Subjetividad y luchas en el umbral de los excesos de justicia.

${ }^{15}$ GOLDBERG, Aviva, SIMMERLING, Mary, FRADER, Joel. Why Nondocumented Residents Should Have Access to Kidney Transplantation: Arguments for Lifting the Federal Ban on Reimbursement. Transplantation, v. LXXXIII, n. 1, 2007, p. 17-20; EINOLLAHI, Behzad et alii. Kidney Transplantation: Is There Any Place for Refugees? Transplantation Proceedings, n. 39, 2007, p. 895-897.

${ }^{16}$ MEZZADRA, NIELSON, op. cit., p. 14.

17 Ibidem.
} 
Por el contrario, busca mostrar que los mecanismos de inclusión diferencial que prohíben la inscripción de extranjeros/migrantes en situación "irregular" a lista de espera para trasplante, pero que posibilitan el trasplante mediante donante vivo, son posibles porque coexisten la preocupación por resguardar los bienes sociales proporcionados por el Estado para los nacionales o extranjeros "residentes" (incluido el acceso a ciertos tratamientos de salud), y la creciente obligación moral de los Estados-nación por el cuidado de la vida en el marco del Ilamado gobierno humanitario. Este doble mandato encuentra sustento en los diferentes usos que adquieren los contenidos relativos al derecho a la salud de la Ley de Migraciones. Con relación a ello, se muestra que las normativas sobre trasplante analizadas recurren a un lenguaje humanitario sustentado en la idea de la protección que, empleado de manera ambigua, cumple el efecto de demarcar la frontera entre legítimos e ilegítimos receptores de órganos. El análisis se apoya en las contribuciones teóricas sobre la legitimidad e ilegitimidad de la salud, la enfermedad y el cuerpo del extranjero/inmigrante (las cuales resultan significativas para pensar el modo en que esta figura emerge como controvertida y susceptible de un tratamiento diferenciado), y en la identificación de ciertos mecanismos que hacen posible el filtro selectivo de la movilidad, entre los que se destacan los procesos de regularización migratoria con su posterior efecto de estratificación en el acceso a derechos.

\section{El extranjero/migrante "enfermo": una figura controvertida}

El tratamiento que recibe la población extranjera/migrante en temas de trasplante de órganos se encuentra condicionado por los requisitos de ingreso, egreso y permanencia definidos en la política migratoria y por las disposiciones que la política de trasplante y la política sanitaria en general establecen para el acceso al tratamiento tanto de personas nacionales como no-nacionales. Las configuraciones locales que adquieren ambos campos se vinculan, a su vez, con discusiones sobre ciertos temas presentes en las agendas regionales e internacionales. En esta intersección, los extranjeros/migrantes emergen como una figura controvertida y susceptible de tratamiento diferenciado.

En Argentina, el derecho a la salud de los extranjeros/migrantes es contemplado en la actual ley de migraciones de diversas maneras. En primer lugar, el art. $8^{\circ}$ establece que el Estado tiene la obligación de proporcionar atención sanitaria, independientemente de la situación administrativa de las personas. También la ley migratoria formaliza la idea de que, si otros Estados no pueden garantizar el acceso de sus nacionales a determinados tratamientos, éstos pueden radicarse temporaria o transitoriamente en Argentina bajo la subcategoría de pacientes bajo tratamientos médicos, para lo cual se deben cubrir determinados requisitos establecidos por la Dirección Nacional de Migraciones (DNM). Por último, reconoce que las personas que invoquen 
razones de salud que a criterio de la DNM hagan presumir riesgo de muerte por falta de tratamiento médico en caso de que fueren obligadas a regresar a su país de origen, pueden radicarse bajo la subcategoría de razones humanitarias ${ }^{18}$. En la Ley de Migraciones se define a "razones humanitarias" como aquellas situaciones que justifiquen a juicio de la DNM un tratamiento especial.

De acuerdo a Fassin, la razón humanitaria ${ }^{19}$ constituye la prueba de humanidad que materializa la obligación de los estados nacionales de cumplir con el doble mandato de actuar en nombre de los derechos de los nacionales y de los extranjeros presentes (o no) en el territorio nacional. El autor plantea que este "despliegue de los sentimientos morales en las políticas contemporáneas ${ }^{\prime 20}$, constituye en la actualidad el fundamento de lo que él denomina gobierno humanitario, el cual que puede ser definido a partir de las dos nociones que comprende, a saber: Gobierno, como el "conjunto de dispositivos establecidos y de las acciones realizadas para administrar, regular y favorecer la existencia de los seres humanos" (que sobrepasa la intervención del Estado nacional, por lo cual es comprendido de manera amplia); y Humanitario, constituido por dos dimensiones de la noción de humanidad: "como el conjunto de seres humanos que comparten una misma condición (pertenecer a la humanidad) y como movimiento afectivo que conduce hacia otros semejantes (dar prueba de humanidad: humanesess $\left.{ }^{21}\right)^{\prime \prime}$. Según el autor, lo que se encuentra en el núcleo del gobierno humanitario es el reconocimiento de la vida como un bien supremo (definido como biolegitimidad ${ }^{22}$ ) y la preocupación por salvar aquellas vidas que se encuentran físicamente amenazadas.

Al ser planteada la razón humanitaria como prerrogativa de la comunidad ética internacional, determinados cuerpos enfermos o sufridos, entre los que se encuentran los cuerpos de los inmigrantes y los refugiados, encontraron una nueva legitimidad allí donde eran considerados una presencia ilegítima. De acuerdo a Sayad, desde la lógica del "balance contable" 23 asociada a la inmigración, la legitimidad o ilegitimidad de los inmigrantes es valorada en función de los beneficios o los costos que su presencia signifique para el Estado y las instituciones de la sociedad receptora. En este marco, la presencia del inmigrante, en particular si éste se ve atravesado por una enfermedad, altera el orden público: "puesto que el inmigrado no tiene sentido y existencia más que a través del trabajo, la enfermedad por sí misma no puede dejar de ser experimentada como la negación del inmigrado" 24 . Con relación a ello, se ha producido un discurso que reconoce a la inmigración como "problema" o asociada a problemas (vinculada, en presencia

\footnotetext{
${ }_{18}^{18}$ Los artículos mencionados se encuentran reglamentados por decreto 616/2010.

19 FASSIN, Didier. La razón humanitaria. Una historia moral del tiempo presente.

20 Ibidem, p. 10.

${ }^{21}$ Ibidem, p. 11.

22 IDEM. Gobernar por los cuerpos, políticas de reconocimiento hacia los pobres y los inmigrantes en Francia.

${ }^{23}$ SAYAD, Abdelmalek. Estado, Nación e Inmigración. El orden nacional ante el desafío de la inmigración.

${ }^{24}$ IDEM. La doble ausencia: De las ilusiones del emigrado a los padecimientos del inmigrado, p. 256.
} 
de la enfermedad, a la discusión sobre los costos de la inmigración) y que cumple con la función de regular una presencia ilegítima ${ }^{25}$.

Fassin considera que las nociones propuestas por Sayad sobre la ilegitimidad del cuerpo enfermo del inmigrante fueron construidas a comienzos de los años ochenta en Francia cuando "la demanda de mano de obra extranjera hacía de la inmigración una necesidad social ${ }^{26}$ ". Pero en los años noventa, debido al aumento de la desocupación, se volvió indeseable la inmigración de mano de obra no calificada y se pusieron en marcha medidas de rechazo por parte de los gobiernos frente al ingreso de aquellos inmigrantes para los que no había demanda en el mercado de trabajo. La representación del trabajador inmigrante legitimado por su capacidad de producción y cuya enfermedad era fuente de sospecha, fue reconfigurada: improductivo para el mundo económico se tornó una preocupación moral para el gobierno humanitario ${ }^{27}$. Acorde con estos cambios el autor sugiere que en la época actual, el cuerpo del extranjero no es el mismo que el cuerpo del inmigrado del cual hablaba Sayad: ahora, el cuerpo del extranjero que aparece como inútil para la economía política, encuentra un nuevo lugar en la economía moral ${ }^{28}$.

Si bien la razón humanitaria permea la legislación sobre el derecho a la salud de los migrantes en Argentina, aquí se sugiere que la legitimidad del cuerpo "enfermo" del extranjero/migrante, que se ha materializado en la ley de migraciones en lo referido a su derecho a la salud, es coexistente a su misma condición de ilegitimidad, aunque esta última no pueda ser percibida de manera inmediata. Diversos autores han señalado que, si bien la ley migratoria reconoce los derechos de los migrantes y la migración como un derecho humano, continúa produciendo distintas categorías y clasificaciones que refuerzan la condicionalidad del ingreso y la permanencia de los extranjeros/migrantes en la sociedad argentina ${ }^{29}$. Pero, ipara qué sería necesario establecer distintos requisitos y categorías de ingreso en lo que respecta al derecho a la salud, si éste ya estaría contemplado en el art $8^{\underline{0}}$ como derecho humano fundamental que no distingue entre extranjeros en situación de "regularidad" o "irregularidad migratoria"? ¿Para qué gestionar este derecho de manera diferenciada?

Como ha sido señalado por López Sala ${ }^{30}$, históricamente los Estados nacionales modernos han participado en la selección de los flujos migratorios

\footnotetext{
${ }^{25}$ Ibidem.

${ }^{26}$ FASSIN, La razón humanitaria..., op. cit., p. 130.

27 Ibidem.

${ }^{28}$ Ibidem.

29 PACECCA, María Inés. Migrantes de ultramar, migrantes limítrofes. Políticas migratorias y procesos clasificatorios. Argentina, 1945-1970; DOMENECH, op. cit.; PIZARRO, Cynthia. Ser boliviano en la región metropolitana de la ciudad de Córdoba. Localización socio-espacial, mercado de trabajo y relaciones interculturales.

${ }^{30}$ LÓPEZ SALA, Ana María. Pasar la línea. El Estado en la regulación migratoria desde una perspectiva comparada.
} 
deseables y no deseables a través de políticas de acceso condicionado y selectivo. Desde ese marco, la práctica de identificación de los denominados receptores legítimos de los bienes colectivos proporcionados por el Estado, una de las funciones constitutivas del control migratorio, ha permitido contener el acceso de aquellos sujetos considerados consumidores de recursos, o de diversas formas de migración abusiva o contraproducente para el interés general ${ }^{31}$ (lo que en términos de Sayad implica la regulación de una presencia ilegítima). En el marco del actual régimen global de control migratorio o globalización del control migratorio ${ }^{32}$, se ha propuesto una forma de gestionar la migración que vincula administración efectiva de la migración y protección de los derechos humanos de los migrantes. Esta perspectiva parte del supuesto (difundido por Bimal Ghosh) de que si se puede ordenar y predecir la migración, ésta será beneficiosa y las ganancias derivadas podrán repartirse entre las naciones de origen y de destino. Para ello se sugirió la armonización de un marco multilateral que administrara cooperativamente la migración internacional, conocido como "nuevo régimen internacional para el movimiento ordenado de personas" o migration management ${ }^{33}$.

En el actual régimen global de control migratorio, los procesos de regularización forman parte de los mecanismos que hacen posible el filtro selectivo de la movilidad ${ }^{34}$, pero su particularidad radica en que la diferenciación entre los flujos migratorios deseados y no deseados se realiza no por medio de un cierre total de las fronteras ${ }^{35}$ y sin que implique necesariamente modalidades represivas ${ }^{36}$. En ese sentido, los procesos de regularización migratoria constituyen las vías para ejercer control sin coerción, o lo que ha sido caracterizado por Domenech como "control con rostro humano" ${ }^{37}$. Con relación a este trabajo, se considera que las categorías y subcategorías de ingreso referidas al derecho a la salud y a la preocupación por el cuidado y preservación de la vida los migrantes contenidas en la ley migratoria, forman parte de procesos de regularización que de acuerdo al modo en que sean conjugados y puestos en relación con diferentes cuestiones o "problemas" planteados en torno a la presencia extranjera/migrante, como veremos a continuación, producen mecanismos y procesos de inclusión diferencial para las personas.

\footnotetext{
31 Ibidem.

32 DÜVELL, Franck. Some Reasons and Conditions for a World Without Immigration Restrictions.

${ }^{33} \mathrm{GHOSH}$, Bimal. Managing Migration: Time for a New International Regime; IDEM. Derechos humanos y migración: el eslabón perdido.

${ }^{34}$ DE GENOVA, Nicholas. Migrant "Illegality" and Deportability in Everyday Life.

${ }^{35}$ DÜVELL, op. cit.; MEZZADRA, Sandro. Capitalismo, migraciones y luchas sociales. La mirada de la autonomía; MEZZADRA, NIELSON, op. cit.

${ }^{36}$ DOMENECH, Eduardo. Las migraciones son como el agua: Hacia la instauración de políticas de "Control con rostro humano".

37 Ibidem. El autor ha señalado que el énfasis en el discurso de los derechos humanos fue lo que le otorgó al paradigma del migration management la legitimidad necesaria para ser aceptado e implementado, pero que en el fondo revelaba la preocupación por el control de la "migración irregular".
} 


\section{La inclusión diferencial como respuesta a la "escasez de órganos" y las "prácticas no éticas"}

El INCUCAI ha determinado que existen "situaciones especiales de inscripción a lista de espera" ${ }^{\prime 38}$, reguladas por resoluciones específicas, entre las que se encuentra la actual Resolución №342/09 que regula la inscripción de personas extranjeras/migrantes a lista de espera para trasplante. Sin embargo, la primera normativa sobre inscripción de extranjeros/migrantes a lista de espera para trasplante fue dictada el 15 de abril de 2004 (tres meses posteriores a la promulgación de la ley migratoria argentina). Entre sus artículos, fijaba diferencias para la inscripción a lista de espera según la situación administrativa de los migrantes y argumentaba:

Es facultad del INCUCAl adoptar los criterios de asignación de órganos y tejidos que permita el acceso de la totalidad de los pacientes a la lista de espera en igualdad de condiciones. Que dichos criterios deben contemplar el aumento de solicitudes de inscripciones de personas extranjeras en las listas de espera y la escasez de órganos para atender las necesidades de los pacientes en su conjunto. Que en este sentido resulta indispensable establecer requisitos que regulen las referidas inscripciones, basándose para ello en principios de equidad, justicia y solidaridad social (...) Que el Consejo Ejecutivo de la Organización Mundial de la Salud recomienda a la 57 a Asamblea, en fecha 22 de enero de 2004 (...) la necesidad de adoptar medidas tendientes a proteger a los grupos más pobres y vulnerables frente al turismo de trasplante y la venta de tejidos y órganos. Que dichas exigencias deben sujetarse a las disposiciones contenidas en la Ley de Migraciones $N^{\circ} 25.871$, que fija las políticas y bases estratégicas en materia migratoria por las que el Estado determina las condiciones necesarias para el ingreso y permanencia de extranjeros en el país. Que la mayoría de los países con programas destinados a incrementar la procuración de órganos para trasplante, han establecido restricciones al acceso de pacientes extranjeros a las listas de espera para asignación de órganos cadavéricos. Que llamado a intervenir el Comité de Bioética del INCUCAI, considera de trascendental importancia que las instituciones responsables de la normatización de bienes escasos promuevan condiciones de justicia local que contemplen las características, necesidades y realidades de la sociedad de pertenencia a la cual se deben (Res. № 85/04, resaltado de la autora).

Con base a los fundamentos descriptos, la normativa disponía que la inscripción a lista de espera por parte de los extranjeros que contasen con residencia permanente se llevaría a cabo según los protocolos médicos vigentes de aquel momento, mientras que quienes contaran con residencia temporaria o transitoria, debían acreditar ingreso por tratamiento médico y un certificado oficial del país de origen que indicase que allí no era posible participar de la

\footnotetext{
${ }^{38}$ Situaciones especiales en lista de espera. Disponible en: < http://www.incucai.gov.ar/index.php/ institucional/legislacion >. Consultado: 10.05.2017.
} 
asignación de órganos o tejidos para implante. También establecía que una vez que los extranjeros con residencia temporaria o transitoria hubiesen cumplido los mencionados requisitos, cada caso sería evaluado en particular por el directorio del INCUCAI, el cual podría efectuar las consultas que considerase pertinentes, para aprobar o no la solicitud de inscripción a lista de espera.

Cinco años después del dictado de la normativa mencionada, se crea la Resolución №342/09 que rige en la actualidad. En ella se acentúan ciertas medidas para evitar la inscripción a lista de espera de extranjeros que probablemente quisiesen sortear las disposiciones del INCUCAI, argumentando haberse encontrado con experiencias afines. Al igual que en la normativa anterior, se determina que únicamente podrán inscribirse en lista de espera aquellos extranjeros que cuenten con residencia de carácter permanente otorgada por la DNM y que no se encuentren incluidos en las listas de espera de sus países de origen. El cambio principal radica, por un lado, en que, mientras que en la resolución anterior las solicitudes de inscripción a listas de espera por parte de residentes temporarios y transitorios eran evaluadas por el Directorio del INCUCAI, con las últimas modificaciones, éstas no son admitidas a priori y son evaluadas como casos excepcionales por el Directorio del INCUCAI (en virtud de la excepciones contempladas por Ley de Trasplante). Por otro lado, sólo se acepta el trasplante con donante vivo para los extranjeros con residencia temporaria y transitoria, para lo cual deben concurrir con un donante o contar con un órgano o tejido proveniente de un banco del exterior. Esto significa que no pueden participar del proceso de asignación de un órgano cadavérico proveniente de un donante argentino o extranjero "residente" en Argentina.

Tres argumentos ratifican lo dispuesto por la normativa del año 2004: la escasez de órganos y tejidos en relación a las necesidades de los pacientes que requieren un implante; el incremento significativo de implantes de órganos y tejidos provenientes de donantes cadavéricos a extranjeros que ingresan al país solo para trasplantarse; y, que "el tratamiento de pacientes que no pertenecen al país o jurisdicción se puede aceptar exclusivamente sino perjudica la capacidad de un país de ofrecer servicios de trasplante a su propia población" ${ }^{\prime 39}$. En el año 2012, se dispone que el ingreso de extranjeros para trasplante con donante vivo en categoría de "residentes temporarios" podrá hacerse en cualquiera de las subcategorías previstas en el art. 230 de la ley migratoria, y en categoría de "residentes transitorios" sólo podrá efectuarse en caso de que el ingreso se realice por medio de la subcategoría de tratamiento médico. Se fundamenta la detección de "numerosos casos en que pacientes han ingresado al país como turistas, entendiéndose que dicha situación contradice el espíritu de la resolución, que ha tenido como objetivo darle un marco legal adecuado al trasplante de órganos

${ }^{39}$ Res. INCUCAI №342/09. 
y tejidos que involucre a pacientes inmigrantes" ${ }^{\prime 40}$. La modificación extiende los requisitos de residencia nombrados a los dadores de órganos.

Ya en la primera normativa sobre trasplante a extranjeros/migrantes, dos dilemas éticos eran planteados: permitir la inscripción a lista de espera a extranjeros "no residentes" supondría desatender, en primer lugar, los criterios de "justicia local"41 y, en segundo lugar, las recomendaciones de diversas organizaciones regionales e internacionales con respecto a la necesidad de proteger a los "grupos vulnerables" del "turismo de trasplantes" y del "comercio de órganos". Frente a estas problemáticas, el Estado argentino responde por medio de mecanismos de inclusión diferencial, cuyo resultado consiste en que, en función del tipo de residencia que los extranjeros/migrantes puedan acreditar, será el tipo de trasplante al que puedan acceder. De acuerdo a ello, es dable considerar que no necesariamente el INCUCAI se sujeta a lo dispuesto en la Ley de Migraciones como se argumenta en la resolución, sino que encuentra en la propia ley migratoria las herramientas necesarias para vehiculizar la intención de restringir la inscripción a lista de espera de extranjeros considerados ilegítimos merecedores de órganos para trasplante.

Si bien los argumentos de las resoluciones sostienen que ésta fue creada con el objetivo de que las personas migrantes que residen en el país puedan ingresar a lista de espera, se deja de lado, como ha advertido el CELS ${ }^{42}$ que hay migrantes que viven en Argentina y que no cuentan con la residencia permanente otorgada por la DNM y que a su vez el INCUCAI no ha accedido a generar otras vías alternativas para certificar la residencia en el territorio nacional ${ }^{43}$. De

$\overline{40}$ Res. INCUCAI №288/12.

${ }^{41}$ De acuerdo a los requisitos de espacio solicitados no resulta posible extenderse en la discusión sobre lo "local" en los procesos de distribución de órganos para trasplante. Al respecto, véase: ELSTER, Jon. Justicia local. De qué modo las instituciones distribuyen bienes escasos y cargas necesarias. Desde la perspectiva teórica adoptada en este trabajo, es posible pensar que los abordajes que identifican las economías morales que los sujetos invierten en cada proceso de distribución de recursos construidos por el Estado como "escasos", no necesariamente consideran el hecho de que los criterios construidos en torno a la distribución de bienes sociales (en este caso órganos para trasplante) dejan supeditado el reconocimiento de derechos a las interpretaciones previas sobre el significado de dichos bienes sociales. Podría pensarse que muchos de los análisis en torno los procesos distributivos de justicia en trasplante, son realizados desde perspectivas sobre justicia sanitaria que se fundamentan en lo que Mezzadra y Nielson (2014) caracterizan como enfoques normativos, legales o gubernamentalizados de las teorías distributivas de la justicia. Según estos autores, las teorías de la justicia dejan afuera la discusión de las fronteras (constitutivas de la definición moderna de la ciudadanía) en el contexto actual de la movilidad humana, por lo cual la relación entre justicia y fronteras hasta ahora se ha centrado en el binarismo inclusión/exclusión.

${ }^{42}$ CELS, Estudio sobre los..., op. cit.; IDEM, Niñez, Migraciones..., op. cit.

${ }^{43}$ De acuerdo al informe del CELS (Estudio sobre los..., op. cit.), en el marco de las acciones desarrolladas por la Mesa de trabajo Interinstitucional en la ciudad de Buenos Aires, en el año 2012 se convocó a funcionarios del INCUCAI a una reunión en la cual se les solicitó y se consensuó con ellos la posibilidad de desarrollar un trabajo articulado con la DNM, poniendo en marcha un protocolo de actuación para aquellos casos de personas migrantes que residen 
allí que la "irregularidad migratoria" sea el producto de decisiones estatales y políticas y no una responsabilidad de las personas ${ }^{44}$.

Además de la escasez de órganos, uno de los principales argumentos que prohíben la inscripción de extranjeros "no residentes" a lista de espera, fue la necesidad de proteger del "turismo de trasplantes" y del "comercio de órganos" a ciertos grupos definidos como vulnerables, en concordancia con las recomendaciones de diferentes organismos vinculados a la actividad de trasplante ${ }^{45}$. En la Declaración de Estambul ${ }^{46}$, el "tráfico de órganos", el "comercio de trasplantes" y el "turismo de trasplantes" fueron considerados "prácticas no éticas":

Los grandes avances científicos y clínicos de entregados profesionales de la salud, así como los numerosos actos de generosidad de los donantes de órganos y sus familias (...) han estado manchados por los numerosos informes sobre el tráfico con seres humanos que se utilizan para extraer órganos y sobre pacientes-turistas de países ricos que viajan al extranjero para comprar órganos a la gente con menores recursos (...) Las prácticas no éticas son, en parte, una consecuencia no deseada de la escasez mundial de órganos para trasplantes (Declaración de Estambul, 2008, p. 1, resaltado de la autora).

El tráfico de órganos y el turismo de trasplantes violan los principios de igualdad, justicia y respeto de la dignidad humana, y deberían prohibirse. Puesto que los donantes con menores recursos económicos o más vulnerables son el blanco de comercialización de trasplantes (...) las prácticas que induzcan a los grupos o individuos vulnerables (como las personas analfabetas y con pocos recursos económicos, los inmigrantes indocumentados, los presos y los refugiados políticos o económicos) a ser donantes vivos son incompatibles con el objetivo de combatir el tráfico de órganos y el turismo y comercialización de trasplantes (Declaración de Estambul, 2008, p. 4, resaltado de la autora).

En el documento citado se sostuvo que la noción "turismo de trasplantes" tiene como antesala el "viaje para trasplantes", definido como "el traslado

efectivamente en Argentina y que no cuentan con la residencia permanente requerida para el ingreso a lista de espera. Sin embargo, a la fecha este compromiso no ha sido concretado.

${ }^{44}$ DE GENOVA, op. cit.; GONZÁLEZ CÁMARA, Noelia. De indeseables a ilegales: Una aproximación a la irregularidad migratoria.

${ }^{45}$ Entre los documentos se señalan: Comité de Bioética del INCUCAI: Sobre la ética de la no comercialización de órganos, 1999; Declaratoria de la RCIDT, Mar del Plata, 2005; Declaratoria de la RCIDT sobre Rechazo al Turismo de Trasplante, 2008; Acuerdo Mercosur № 05/2009. Combate al tráfico de órganos, tejidos y células y al turismo de trasplantes en los Estados partes y asociados al Mercosur, 2009; Declaración de Estambul sobre el combate al Tráfico de Órganos y Turismo de trasplante. Cumbre internacional sobre turismo de trasplantes y tráfico de órganos. Estambul, Turquía, 2008.

${ }^{46}$ La Declaración de Estambul fue elaborada por profesionales de la actividad trasplantológica en la Cumbre Internacional sobre Turismo de Trasplantes y Tráfico de Órganos desarrollada en Turquía en el año 2008. En coincidencia con Amahazion (2015), aunque la declaración no es vinculante, señala el consenso de la comunidad médica mundial con relación al problema de los trasplantes comerciales. 
de órganos, donantes, receptores o profesionales del trasplante fuera de las fronteras jurisdiccionales dirigido a realizar un trasplante". El "viaje para trasplantes" se convierte en "turismo de trasplantes" si implica el "tráfico de órganos" o la "comercialización de trasplantes"47, o si los recursos (órganos, profesionales y centros de trasplantes) dedicados a suministrar trasplantes a pacientes de otro país debilitan la capacidad del país de ofrecer servicios de trasplantes a su propia población" ${ }^{\prime 48}$. Es posible pensar que en la definición de "turismo de trasplantes", son comprendidos dos fenómenos diferentes: por un lado, el "comercio de trasplantes" y/o "tráfico de órganos" y, por el otro, el destino de recursos (en este caso órganos y servicios de trasplantes) a extranjeros no residentes. Estos fenómenos que corresponden a órdenes distintos (ya que "comercio de trasplantes" y "tráfico de órganos" podrían ser abordados desde enfoques criminológicos, mientras que la cuestión de la distribución de recursos escasos correspondería quizás a debates de ética y justicia), son englobados en una misma definición como únicas opciones posibles.

Esta ambigüedad del concepto no es ajena a las consecuencias que produce, porque precisamente, en la existencia del $o$ radica la potencialidad de la categoría, la cual nos enfrenta a un falso dilema. En otras palabras, o estamos frente a "tráfico de órganos" y/o "comercio de trasplante", o estamos frente a la imposibilidad de trasplantar a un nacional, porque en su lugar se destinaron recursos para un extranjero. La primera de las alternativas constituiría un delito y, la segunda, una práctica ilegítima y potencialmente criminalizable. Desde este punto de vista, la presencia de un extranjero "no residente" podrá siempre ser cuestionada, puesta en duda, porque constituye, como ha sostenido Sayad, una presencia ilegítima ${ }^{49}$. Esto permite pensar que el proceso de categorización ${ }^{50}$ presente en la construcción de la figura del migrante "irregular" como un "turista de trasplante" podría constituir una técnica de securitización de la migración que permite condenar a los extranjeros incluso antes de que hayan hecho nada, anticipando perfiles de riesgo y generalizando potenciales conductas en función de esta categoría construida ${ }^{51}$. Esto se expresa en la medida en que, a partir de la construcción anticipada de la sospecha, se habilita la solicitud de regularización migratoria para acceder a lista de espera de trasplante.

\footnotetext{
47 Para las definiciones de "tráfico de órganos" y "comercio de trasplantes" véase: Declaración de Estambul sobre el combate al Tráfico de Órganos y Turismo de trasplante.

48 Ibidem.

49 SAYAD, La doble ausencia..., op. cit.

${ }^{50}$ BIGO, Didier. Security and Inmigration: Toward a Critique of the Governmentality of Unease.

${ }^{51}$ Al referirse a la securitización de la migración, Bigo (ibidem) señala que en el proceso de construcción de una sociedad peligrosa y de riesgo, la inmigración (al ser vinculada al terrorismo, al crimen, al desempleo y a la discusión sobre la ciudadanía) emerge como una de las nuevas amenazas a la seguridad. Específicamente sobre la construcción de la migración irregular como una de las nuevas amenazas a la seguridad véase: DALMASSO, Clara. ¿Quiénes son terroristas? La migración irregular y forzada como nuevas amenazas a la seguridad en el cono sur. Astrolabio, n. 17, 2016, p. 114-145.
} 
En los documentos analizados el migrante "irregular" no es identificado exclusivamente como "turista de trasplante", sino como posible "víctima" (bajo la figura del indocumentado o refugiado) de quienes lleven adelante "prácticas no éticas". Ese desplazamiento en la categoría del migrante "irregular" en dos sentidos diferentes, coloca en su centro la noción de protección, pero, como propone Bigo $^{52}$, es preciso interrogarse sobre quién o qué es objeto de protección, para poder identificar los diferentes significados del término y las direcciones en las cuales la acción de la protección puede moverse. De acuerdo al autor, la protección puede significar desde ayudar a alguien hasta detenerlo o vigilarlo, y siempre supone una relación asimétrica en la que intervienen el enemigo, el protector y el protegido. En el caso en estudio, el protegido podría verse representado por las personas inscriptas en lista de espera, los potenciales receptores (nacionales y extranjeros "residentes") de órganos para trasplante, y las personas "vulnerables" al "turismo de trasplantes" y al "comercio de órganos". El enemigo aparece identificado bajo la figura del "turista de trasplante", y el INCUCAI encarna el rol del protector, como organismo responsable y representante del "bien público" ${ }^{53}$ en materia de trasplantes. La construcción de la sospecha que envuelve al migrante "irregular" en ambas situaciones es plasmada en una normativa que dice "proteger a todos": a las víctimas de las "prácticas no éticas", a los nacionales y "residentes" inscriptos en lista de espera, a los "avances de entregados profesionales de la salud".

\section{Consideraciones finales}

A lo largo de este artículo se pretendió mostrar que la noción de inclusión diferencial puede resultar apropiada para comprender los grados de subordinación y reglas a los que los extranjeros/migrantes en situación administrativa "irregular" están ligados en la política de trasplantes. En ese sentido, se evidenciaron procesos de cierre y apertura de fronteras en la medida en que la inscripción a lista de espera para recibir órganos de donantes cadavéricos quedó reservada para los extranjeros/migrantes con residencia "permanente", mientras que aquellos que no pudiesen acreditar dicho tipo de residencia, pueden ser trasplantados mediante donante vivo si acreditan, por ejemplo, ingreso por tratamiento médico. En este sentido, se consideró que si bien este mecanismo posibilita el acceso al tratamiento a ciertas personas (aunque de modo diferenciado) actúa como una herramienta de control frente a la posible movilidad en condiciones "irregulares" en búsqueda de trasplante. Lo significativo es que estas prácticas de control, son definidas a partir del valor del cuidado y preservación de la vida humana.

\footnotetext{
52 IDEM. Protection. Security, territory and population.

${ }^{53}$ BOURDIEU, Pierre. Sobre el Estado. Cursos en el Collège de France (1989-1992).
} 
Lo abordado sugiere que, desde el discurso de la protección la normativa sobre trasplante a extranjeros termina produciendo el efecto de excluir a muchos migrantes de la posibilidad de participar en una lista de espera en un país diferente al de origen. En este sentido, el discurso oficial enfatiza la necesidad de protección del extranjero en situación "irregular" (en caso de convertirse en víctima como potencial donante en el mercado ilegal de comercialización de órganos y del "turismo de trasplantes"), acentúa su criminalización al momento de identificarlo como posible comprador de órganos o "turista de trasplante" y en el medio valida su desprotección en caso de constituirse en potencial receptor en el campo legal de la inscripción a lista de espera. El análisis propuesto considera que el "turismo de trasplante", al cual se alude como argumento de las medidas analizadas, más que interrogado como un fenómeno en sí mismo, requiere ser problematizado en cuanto categoría analítica, en sintonía con aquellas sugerencias de Bourdieu sobre someter todas las categorías estatales a "una duda radical".

\section{Bibliografía}

BIGO, Didier. Protection. Security, territory and population. In HUYSMANS, Jef; DOBSON, Andrew; PROKHOVINK, Raia. The politics of Protection. Sites of insecurity and political agency. London and New York: Routledge, 2006, p. 84-100.

BIGO, Didier. Security and Inmigration: Toward a Critique of the Governmentality of Unease. Alternatives, n. 22, 2002, p. 63-92.

BOURDIEU, Pierre. Sobre el Estado. Cursos en el Collège de France (1989-1992). Barcelona, España: Editorial Anagrama, 2014.

DE GENOVA, Nicholas. Migrant "Illegality" and Deportability in Everyday Life. Annual Review of Anthropology, n. 31, 2002, p. 419-447.

Decreto № 616/2010, sobre Reglamentación de la Ley de Migraciones № 25.871 y sus modificatorias. Boletín Oficial № 31.898. 6 de mayo de 2010.

DOMENECH, Eduardo. La visión estatal sobre las migraciones en la Argentina reciente. De la retórica de la exclusión a la retórica de la inclusión. In DOMENECH, Eduardo. Migración y política: el Estado interrogado. Procesos actuales en Argentina y Sudamérica. Córdoba: Universidad Nacional de Córdoba, 2009, p. 21-70.

DOMENECH, Eduardo. Las migraciones son como el agua: Hacia la instauración de políticas de "control con rostro humano. La gobernabilidad migratoria en la Argentina. POLIS, Revista Latinoamericana, n. 35, 2013, p. 1-17.

DÜVELL, Franck. Some Reasons and Conditions for a World Without Immigration Restrictions. ACME: An International E-Journal for Critical Geographies, v. 2, n. 2, 2003, p. 201-209.

FASSIN, Didier. Gobernar por los cuerpos, políticas de reconocimiento hacia los pobres y los inmigrantes en Francia. Cuadernos de Antropología Social, n. XVII, 2003, p. 49-78.

FASSIN, Didier. La razón humanitaria. Una historia moral del tiempo presente. Buenos Aires: Prometeo, 2016. 
GHOSH, Bimal. Derechos humanos y migración: el eslabón perdido. Migración y Desarrollo, n. 10, 2008, p. 37-62.

GHOSH, Bimal. Managing Migration: Time for a New International Regime. Oxford: Oxford University Press, 2000.

GONZÁLEZ CÁMARA, Noelia. De indeseables a ilegales: Una aproximación a la irregularidad migratoria. ARBOR Ciencia, Pensamiento y Cultura, n. 744, 2010, p. 671-687.

INCUCAI, Ministerio de Salud. Resolución № 288/2012 Inscripción de ciudadanos extranjeros en lista de espera.

INCUCAI, Ministerio de Salud. Resolución № 342/2009 Inscripción de ciudadanos extranjeros en lista de espera.

INCUCAI, Ministerio de Salud. Resolución № 85/2004 Inscripción de ciudadanos extranjeros en lista de espera.

Ley de Migraciones № 25.871.

LÓPEZ SALA, Ana María. Pasar la línea. El Estado en la regulación migratoria desde una perspectiva comparada. RIFP, n. 27, 2006, p. 71-100.

MEZZADRA, Sandro; NIELSON, Brett. Fronteras de Inclusión diferencial. Subjetividad y luchas en el umbral de los excesos de justicia. Papeles del CEIC, v. II, 2014, p. 1-30.

MEZZADRA, Sandro. Capitalismo, migraciones y luchas sociales. La mirada de la autonomía. Nueva Sociedad, n. 237, 2012, p. 159-178.

PACECCA, María Inés. Migrantes de ultramar, migrantes limítrofes. Políticas migratorias y procesos clasificatorios. Argentina, 1945-1970. Informe final del concurso: Culturas e identidades en América Latina y el Caribe. Programa Regional de Becas CLACSO, 2001.

PIZARRO, Cynthia. Ser boliviano en la región metropolitana de la ciudad de Córdoba. Localización socio-espacial, mercado de trabajo y relaciones interculturales. Córdoba: EDUCC, 2011.

SAYAD, Abdelmalek. Estado, Nación e Inmigración. El orden nacional ante el desafío de la inmigración. Apuntes de Investigación/Tema central: Partir, 2008, p. 100-116. SAYAD, Abdelmalek. La doble ausencia. De las ilusiones del emigrado a los padecimientos del inmigrado. Barcelona: Anthropos, 2010.

Recibido para publicación en 31.05.2017 Aceptado para publicación en 26.06.2017 Received for publication in May 315t, 2017 Accepted for publication in June $26^{\text {th }}, 2017$ 